\title{
Pembrolizumab-Induced Adrenal Insufficiency in Patients with Untreated Advanced Non-Small Cell Lung Cancer: A Case Series
}

\author{
Kei Sonehara Kazunari Tateishi Taisuke Araki Masamichi Komatsu \\ Jumpei Akahane Hiroshi Yamamoto Masayuki Hanaoka
}

First Department of Internal Medicine, Shinshu University School of Medicine, Matsumoto, Japan

\section{Keywords}

Adrenal insufficiency · Immune-related adverse events · Non-small cell lung cancer .

Pembrolizumab

\section{Abstract}

Pembrolizumab-induced adrenal insufficiency (Al) is recognized as a rare immune-related adverse event (irAE) that can be fatal if diagnosis is delayed. Clinical features of $\mathrm{Al}$ in patients with advanced non-small cell lung cancer (NSCLC) who received pembrolizumab as the firstline treatment were observed. Five out of 49 patients with untreated advanced NSCLC developed Al between April 2017 and February 2021. Of the 5 patients, 4 developed Al with a grade $>3$ and were hospitalized. The median time of the onset of Al from the start of first-line treatment was 4.63 months. All the patients improved after corticosteroid replacement therapy. The efficacy of treatment was complete response in 1 patient and partial response in 4 patients. Median progression-free survival was 37.7 months, and overall survival had not been reached. This case series revealed the efficacy of immunotherapy in $\mathrm{Al}$ as an irAE. 


\section{Introduction}

Anti-programmed cell death protein-1 (PD-1) antibody is an immune checkpoint inhibitor (ICI) and is one of the main drug treatments for advanced non-small cell lung cancer (NSCLC). Pembrolizumab and nivolumab are examples of anti-PD-1 antibodies. ICIs are known to cause immune-related adverse events (irAEs), especially in the skin, lungs, and endocrine and digestive systems. Endocrine irAEs include hypothyroidism, hyperthyroidism, hypophysitis, and adrenal insufficiency (AI) [1]. The incidence of pembrolizumab-induced AI is $0.98 \%$, the symptoms are nonspecific, and the severity rate is high [2]. Herein, we report the cases of 5 patients who developed AI after receiving pembrolizumab as the firstline treatment. We also describe the association between the efficacy of immunotherapy and the clinical features of AI.

\section{Case Presentation}

All patient-related data were retrospectively collected from the electronic medical records of Shinshu University Hospital, in accordance with the principles of the Declaration of Helsinki. The best objective response to first-line treatment was evaluated using the Response Evaluation Criteria in Solid Tumors (version 1.1) [3]. Progression-free survival (PFS) was defined as the start of first-line treatment to the date of progressive disease or to the date of death, while overall survival (OS) was measured from the start of first-line treatment to the date of the last follow-up. IrAE was graded according to Common Terminology Criteria for Adverse Events, version 5.0.

A total of 49 patients with NSCLC who received pembrolizumab as the first-line treatment between April 2017 and February 2021 were included in our case series. The cutoff date for follow-up was June 30, 2021. Forty-one patients were male and 8 were female, with a median age of 72 years (range: 40-87 years). Twenty-three patients received pembrolizumab monotherapy as first-line treatment, 16 received carboplatin plus pemetrexed plus pembrolizumab, 7 received carboplatin plus nab-paclitaxel plus pembrolizumab, and 3 received carboplatin plus paclitaxel plus pembrolizumab. We defined AI as a cortisol level $<4 \mu \mathrm{g} / \mathrm{dL}$ in the morning or $\geq 4 \mu \mathrm{g} / \mathrm{dL}$ and $<18 \mu \mathrm{g} / \mathrm{dL}$ in the morning, responsive to corticosteroid replacement therapy. A total of 5 patients developed AI, and the patient characteristics and efficacy of firstline treatment are shown in Table 1. Of the 5, 3 were male, while 2 were female, with a median age of 63 years (range: 49-79 years). All the patients had good performance status (PS). Two patients received pembrolizumab monotherapy, while 3 received combination therapy, in which pembrolizumab and cytotoxic agents were combined. Table 2 shows the summary of the clinical course in the 5 patients with AI. All the patients were diagnosed with AI after admittance of any symptom. The major complaints were nonspecific symptoms such as anorexia, malaise, fever, diarrhea, and difficulty in movement. In case 4, where the patient developed AI of grade 4, weight loss of $10 \mathrm{~kg}$ or more was observed relative to the time of diagnosis. No significant weight loss was observed in the other 4 patients. Eosinophilia $(>500$ eosinophils $/ \mu \mathrm{L}$ ) was observed in 1 patient (Case 3). At the onset of AI, 4 out of the 5 patients had poor PS ( $\geq 3$ ) and required hospitalization. The median time of the onset of AI was 4.63 months (range: 2.13-16.13 months). All patients received corticosteroid replacement therapy, and their symptoms improved. Three of the 5 patients discontinued pembrolizumab, but after improvement of AI, 2 of these patients resumed pembrolizumab. In evaluating for survival, the median follow-up was 17.5 months (range: 10.7-48.0 months). The median PFS was 37.7 months, while the OS was not reached. Regarding the best objective response, 1 patient had complete response, and 4 patients had partial response.

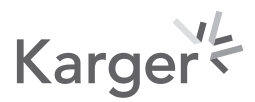


Sonehara et al.: Pembrolizumab-Induced Adrenal Insufficiency as an Immune-Related Adverse Event

Table 1. Clinical characteristics before the initiation of first-line treatment in patients who later developed adrenal insufficiency

\begin{tabular}{ll}
\hline Category & $N$ \\
\hline Patients & 5 \\
Median age (range), years & 63 (49-79) \\
Gender, male/female & $3 / 2$ \\
ECOG performance status, 0/1 & $2 / 3$ \\
Smoking history, current plus former/never & $4 / 1$ \\
Histologic subtype, adeno/squamous/not otherwise specified & $3 / 1 / 1$ \\
Clinical stage (TNM eighth), IVA/IVB/others & $5 / 0 / 0$ \\
Driver mutation, positive/negative & $0 / 5$ \\
PD-L1 TPS, $\geq 50 / 1-49 /<1, \%$ & $3 / 2 / 0$ \\
First-line treatment & \\
Pembrolizumab monotherapy & 2 \\
Carboplatin plus pemetrexed plus pembrolizumab & 1 \\
Carboplatin plus nab-paclitaxel plus pembrolizumab & 2 \\
Best overall response, complete response/partial response & $1 / 4$ \\
Median progression-free survival (95\% CI), months & 37.7 (na-na) \\
Median overall survival (95\% CI), months & na (na-na) \\
\hline
\end{tabular}

ECOG, Eastern Cooperative Oncology Group; TNM, Tumor Nodes Metastasis; PD-L1, programmed cell death ligand-1; TPS, Tumor Proportion Score; CI, confidence interval.

\section{Discussion/Conclusion}

This case series describes the clinical features of pembrolizumab-induced AI in patients with untreated advanced NSCLC. According to World Health Organization's VigiBase, 58\% of patients who develop AI were males. The median age of patients with AI was 66 years, and median time of the onset of AI was 120 days from the start of treatment [4]. In 1 retrospective study, 6 of the 10 patients who developed AI were male. The median age of patients with AI was 66 years, and median time of the onset of AI was 5.6 months [5]. The Japanese adverse drug event report database demonstrated the median time of the onset of nivolumab- and pembrolizumab-induced AI to be 156 and 118 days, respectively [6]. Patient characteristics of AI in our case series were similar to those in these previous studies. But compared with earlier reports, $10.2 \%$ of all patients in this case series were diagnosed with AI more frequently. However, this was considered to be a coincidence because of the small sample size and singlecenter nature of this study [7].

Symptoms of AI such as irAEs are nonspecific and difficult to diagnose, with nearly half being severe at the time of diagnosis. In our case series, 4 of the 5 patients had severe AI as an irAE and were hospitalized, but corticosteroid replacement therapy improved PS in all patients. A previous study showed that high-dose glucocorticoids for the treatment of ipilimumab-induced hypophysitis in patients with melanoma were associated with poor survival [8]. However, in our case series, immunotherapy was effective in patients who developed AI and resulted in prolonged survival. In case 4, pembrolizumab was still discontinued after improvement of grade $4 \mathrm{AI}$, but had maintained CR for about 6 months [9]. In cases $1,2,3$, and 4, immunotherapy was continued with corticosteroid replacement therapy, and the efficacy of pembrolizumab was good. Twenty milligrams of intravenous hydrocor- 
Sonehara et al.: Pembrolizumab-Induced Adrenal Insufficiency as an Immune-Related Adverse Event

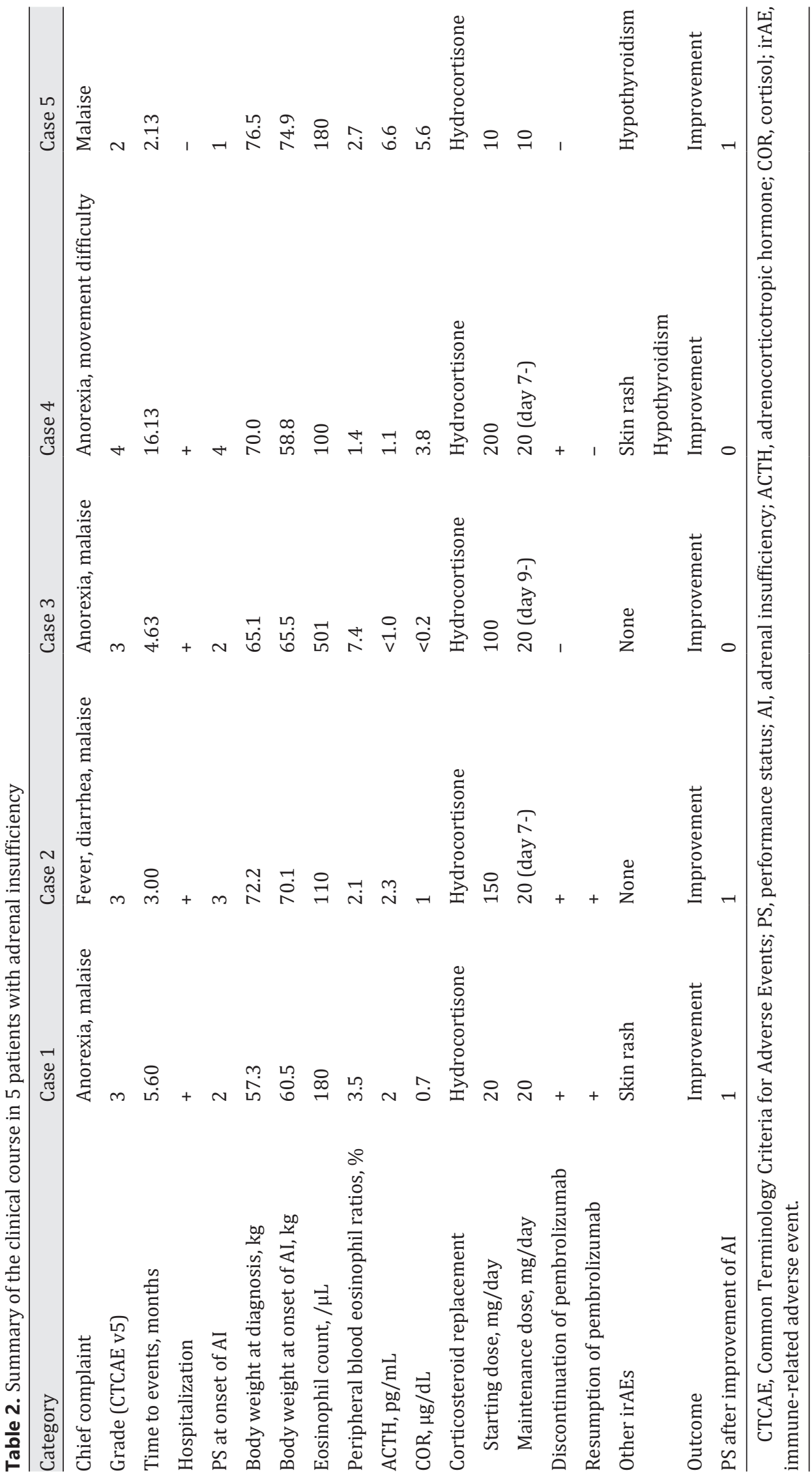


tisone, which is equivalent to $5 \mathrm{mg}$ of oral prednisolone, was administered daily to 4 out of 5 patients. The dose of the bioactive hydrocortisone was insufficient to interfere with the effect of ICI. Ida et al. [5] reported that the median OS in pretreated NSCLC patients while continuing ICIs after the development of AI was 15.4 months, and survival was favorable. Further studies are needed to evaluate the association between steroid dose and the efficacy of ICIs.

Prognostic biomarkers, such as the lung immune prognostic index and neutrophil-tolymphocyte ratio, are well-known factors used to measure the efficacy of ICIs $[10,11]$. In recent years, several studies have reported that the development of irAE was associated with good prognosis in NSCLC patients who received ICIs $[12,13]$. In a multivariate analysis of NSCLC patients who were treated with nivolumab, Haratani et al. [12] demonstrated that the development of irAEs was positively associated with survival outcome, with hazard ratios of 0.525 for PFS and 0.282 for OS. In another retrospective study in our institute, the median PFS and OS were 6.8 and 37.8 months, respectively, in the pretreated NSCLC patients with irAEs and 1.9 and 8.1 months, respectively, in those without irAEs. The median PFS and OS in those with irAEs were significantly longer than those without irAEs (PFS: $p<0.001$ and OS: $p<0.001$ ) [13]. In the present case series, 5 patients were alive at the data cutoff date, and 3 out of 5 patients had other irAEs. A previous report states that among patients who developed irAEs, the incidence of developing multiple irAEs was significantly higher in the ICI-responder group than in the ICInonresponder group ( $41.7 \%$ vs. $0.0 \%, p=0.009$ ) [13]. The good prognosis in our cases might have been due to the absence of distant metastasis other than lung metastasis. Moreover, it was also suggested that the development of AI may be a favorable prognostic factor associated with survival. Further examination and analysis of cases are needed to verify this finding.

In conclusion, we presented the clinical characteristics of pembrolizumab-induced $\mathrm{AI}$ in patients with untreated advanced NSCLC. For patients with AI, corticosteroid replacement therapy would be useful in continuing treatment without diminishing the efficacy of ICIs. This case series suggested that the development of AI as an irAE may be associated with the efficacy of immunotherapy.

\section{Statement of Ethics}

Written informed consent to participate in this study was obtained in the form of opt-out on the website. This study protocol and consent procedure was reviewed and approved by the Research Ethics Board of Shinshu University School of Medicine, Approval No. 4772. Written informed consent was obtained from all patients for publication of this case report and any accompanying images. All data were conducted in accordance with the principles of the Declaration of Helsinki.

\section{Conflict of Interest Statement}

The authors have no conflicts of interest to declare.

\section{Funding Sources}

The authors did not receive any funding.

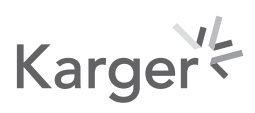




\section{Case Reports in Oncology}

Case Rep Oncol 2021;14:1561-1566

\begin{tabular}{l|l}
\hline DOI: 10.1159/000519597 & ( 2021 The Author(s). Published by S. Karger AG, Basel
\end{tabular} www.karger.com/cro

Sonehara et al.: Pembrolizumab-Induced Adrenal Insufficiency as an Immune-Related Adverse Event

\section{Author Contributions}

K.S. and K.T. drafted the manuscript. K.T. reviewed the manuscript. K.S., K.T., T.A., M.K., T.A., H.Y., and M.H. collected the data.

\section{Data Availability Statement}

All data generated or analyzed during this study are included in this article. Further enquiries can be directed to the corresponding author.

\section{References}

1 Corsello SM, Barnabei A, Marchetti P, De Vecchis L, Salvatori R, Torino F. Endocrine side effects induced by immune checkpoint inhibitors. J Clin Endocrinol Metab. 2013;98:1361-75.

2 Wang PF, Chen Y, Song SY, Wang TJ, Ji WJ, Li SW, et al. Immune-related adverse events associated with antiPD-1/PD-L1 treatment for malignancies: a meta-analysis. Front Pharmacol. 2017;8:730.

3 Eisenhauer EA, Therasse P, Bogaerts J, Schwartz LH, Sargent D, Ford R, et al. New response evaluation criteria in solid tumours: revised RECIST guideline (version 1.1). Eur J Cancer. 2009;45:228-47.

4 Grouthier V, Lebrun-Vignes B, Moey M, Johnson DB, Moslehi JJ, Salem JE, et al. Immune checkpoint inhibitorassociated primary adrenal insufficiency: WHO VigiBase Report Analysis. Oncologist. 2020;25:696-701.

5 Ida H, Goto Y, Sato J, Kanda S, Shinno Y, Morita R, et al. Clinical characteristics of adrenal insufficiency as an immune-related adverse event in non-small-cell lung cancer. Med Oncol. 2020;37:30.

6 Hasegawa S, Ikesue H, Nakao S, Shimada K, Mukai R, Tanaka M, et al. Analysis of immune-related adverse events caused by immune checkpoint inhibitors using the Japanese Adverse Drug Event Report database. Pharmacoepidemiol Drug Saf. 2020;29:1279-94.

7 Ariyasu R, Horiike A, Yoshizawa T, Dotsu Y, Koyama J, Saiki M, et al. Adrenal insufficiency related to antiprogrammed death-1 therapy. Anticancer Res. 2017;37(8):4229-32.

8 Faje AT, Lawrence D, Flaherty K, Freedman C, Fadden R, Rubin K, et al. High-dose glucocorticoids for the treatment of ipilimumab-induced hypophysitis is associated with reduced survival in patients with melanoma. Cancer. 2018;124:3706-14.

9 Sonehara K, Tateishi K, Hirabayashi T, Araki T, Ikuyama Y, Machida R, et al. A case of lung adenocarcinoma with long-term response after late-onset pembrolizumab-induced acute adrenal insufficiency. Case Rep Oncol. 2021 Jan-Apr;14(1):1-7.

10 Mezquita L, Auclin E, Ferrara R, Charrier M, Remon J, Planchard D, et al. Association of the lung immune prognostic index with immune checkpoint inhibitor outcomes in patients with advanced non-small cell lung cancer. JAMA Oncol. 2018;4(3):351-7.

11 Diem S, Schmid S, Krapf M, Flatz L, Born D, Jochum W, et al. Neutrophil-to-lymphocyte ratio (NLR) and plateletto-lymphocyte ratio (PLR) as prognostic markers in patients with non-small cell lung cancer (NSCLC) treated with nivolumab. Lung Cancer. 2017;111:176-81.

12 Haratani K, Hayashi H, Chiba Y, Kudo K, Yonesaka K, Kato R, et al. Association of immune-related adverse events with nivolumab efficacy in non-small-cell lung cancer. JAMA Oncol. 2018;4(3):374-8.

13 Sonehara K, Tateishi K, Araki T, Komatsu M, Yamamoto H, Koizumi T, et al. The role of immune-related adverse events in prognosis and efficacy prediction for patients with non-small cell lung cancer treated with immunotherapy: a retrospective clinical analysis. Oncology. 2021;99(5):271-9. 